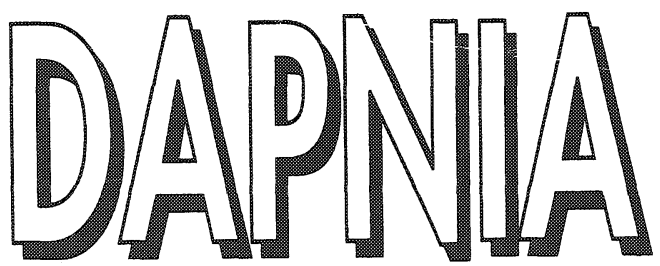

CERN LIBRARIES, GENEVA

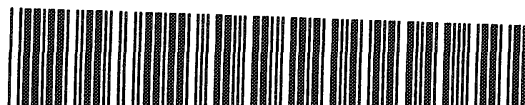

CM-P00058557

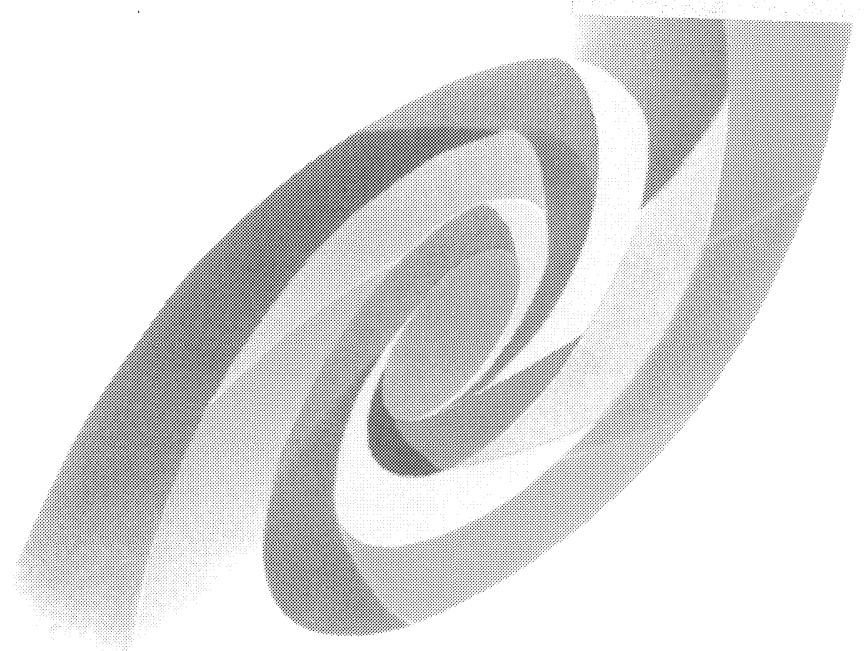

DAPNIA-05-277

$10 / 2005$

\title{
Polarization in QCD
}

Jean-Marc Le Goff

22nd International Symposium On Lepton-Photon Interactions At High Energy (LP 2005), Uppsala (sweden), June 30-July 5, 2005

Département d'Astrophysique, de Physique des Particules, de Physique Nucléaire et de l'Instrumentation Associée DSM/DAPNIA, CEA/Saclay F - 91191 Gif-sur-Yvette Cédex

Tél : (1) 69082402 Fax : (1) 69089989

http : //www-dapnia.cea.fr 


\title{
POLARIZATION IN QCD
}

\author{
J.-M. LE GOFF \\ DAPNIA/SPhN CEA-Saclay, F91191 Gif-sur-Yvette, France \\ E-mail: jmlegoff@cea.fr
}

\begin{abstract}
We first deal with the muon anomalous magnetic moment which is found to differ from the standard model prediction by $2.7 \sigma$. A new proposal will aim at reducing the error by a factor 2 . The main part of the paper then deals with the spin structure of the nucleon which can be studied in terms of quark and gluon helicity distributions, quark transversity distributions and generalized parton distributions. The main recent results are first indications that the total gluon spin in the nucleon might be small and a first measurement of the Collins fragmentation function which is needed to extract transversity distributions from semi-inclusive DIS data.
\end{abstract}

\section{The muon anomalous magnetic} moment

The muon anomalous magnetic moment $a_{\mu}=$ $\left(g_{\mu}-2\right) / 2$ can be both measured and computed very accurately. Deviations from the standard model prediction are expected according to many models: SUSY, leptoquark, muon substructure or anomalous $\mathrm{W}$ coupling.

The E821 experiment ${ }^{1}$ in Brookhaven makes use of a muon beam obtained by pion weak decay. This beam is naturally polarized due to parity violation in pion decay. The muons are injected in a ring where their spins rotate slightly faster than their momentum. This precession is proportional to $a_{\mu}$. The rate of positrons from muon decay is recorded in 24 electromagnetic calorimeters. Due to parity violation in muon decay this rate is a decreasing exponential modulated at the precession frequency, as illustrated in Fig. 1. The rate is fitted by $N(t)=N_{0} e^{-t / \tau}[1+$ $\left.A \cos \left(\omega_{a} t+\phi\right)\right]$ which provides the precession frequency $\omega_{a}$. The magnetic field is measured by a NMR probe in units of the free proton precession frequency, $\omega_{p}$. Knowing the ratio of muon-to-proton magnetic moment from muonium experiments, the ratio $\omega_{a} / \omega_{p}$ provides $a_{\mu}=(11,659,208 \pm 5 \pm 3) \times 10^{-10}$. This is an accuracy of $0.5 \mathrm{ppm}$, which is 15 times better than the previous experiment.

The calculation of $a_{\mu}$ in the standard model involves several contributions dis-
Table 1. World averaged experimental measurement and various theoretical contributions to $a_{\mu}$ in units of $10^{-10}$, see text

\begin{tabular}{|l|r|}
\hline QED & $11,659,471.94 \pm 0.14$ \\
Had LO & $693.4 \pm 6.4$ \\
Had LBL & $12.0 \pm 3.5$ \\
Had HO & $-10.0 \pm 0.6$ \\
weak & $15.4 \pm 0.22$ \\
\hline total & $11,659,182.7 \pm 7.3$ \\
exp & $11,659,208 \pm 6$ \\
\hline exp-the & $25.3 \pm 9.4$ \\
\hline
\end{tabular}

played in table 1. The QED contribution is by far the largest. It is computed up to 4 loops and is then very accurately known. The weak contribution is small; it is computed to 2 loops and is also very accurately known. The hadronic contribution is split into three terms: leading order (LO), light by light term (LBL) and higher order (HO). The evaluation of the leading order term (hadronic vacuum polarization) is based on $e^{+} e^{-}$data. An evaluation from tau decay data is also possible but it requires several delicate corrections and it does not give a result compatible with those from $e^{+} e^{-}$.

As can be seen in the table, the world averaged experimental result differ from the standard model prediction by $25.3 \pm 9.4$, i.e. $2.7 \sigma$. A new experiment, E969, is proposed in order to reach $\delta a_{\mu} \approx 2 \cdot 10^{-10}$. The main ideas and the ring will be kept, the num- 


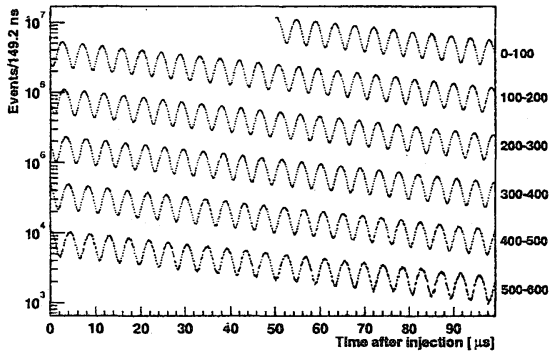

Figure 1. The positron rate in E821 as a function of time exhibits an exponential shape (note logarithmic vertical scale) modulated at the precession frequency.

ber of muons will be increased by a factor 5 and the systematics decreased. Together with expected improvements in the theory a gain by a factor 2 is expected in the error on the difference between experiment and theory. This should clarify whether the $2.7 \sigma$ discrepancy observed is a real effect or not.

\section{Quark polarized distributions}

The spin $1 / 2$ of the nucleon can be decomposed in the contributions from its constituents as ${ }^{2}$

$$
\frac{1}{2}=\frac{1}{2} \Delta \Sigma+\Delta G+L_{q}+L_{g}
$$

where $\Delta \Sigma$ is the total number of quarks with spin parallel to the spin of the nucleon minus the number of quarks with spin antiparallel; once weighted by the spin of the quark, i.e. $1 / 2$, this is the contribution from the quark spins to the nucleon spin. Similarly, $\Delta G$ is the contribution from the spin of the gluons (which have spin 1). Finally, $L_{q}$ and $L_{g}$ are the contributions from the orbital angular momentum of quarks and gluons, respectively.

The contribution $\Delta \Sigma$ can be measured in inclusive deep inelastic scattering (DIS) experiments where a high energy lepton scatters on a nucleon target through the exchange of a virtual photon. There exist only two independent Lorentz invariants which can be chosen as $Q^{2}=-q_{\mu}^{2}$, the virtuality of the photon, and $x_{b j}=Q^{2} / 2 M \nu$, where $\nu$ is the photon energy and $M$ the nucleon mass. $Q^{2}$ gives the probe resolution, while $x_{b j}$ can be interpreted as the fraction of the nucleon momentum carried by the quark which absorbed the virtual photon. In unpolarized DIS one measures cross sections which involve two structure functions depending only on the two invariants $x$ and $Q^{2}$. To first order (i.e. in the parton model) the $Q^{2}$ dependence vanishes, indicating scattering on point-like particles (the quarks) and we have e.g. $F_{1}(x)=\frac{1}{2} \sum e_{q}^{2} q(x)$. Here the quark distribution function $q(x)$ gives the probability density for finding inside the nucleon a quark of flavor $q$ and momentum fraction $x$.

In polarized DIS one measures crosssection spin differences, $\Delta \sigma=\sigma^{\uparrow \downarrow}-\sigma^{\uparrow \uparrow}$, which involve the polarized structure functions $g_{1}$ and $g_{2}$. In the parton model $g_{1}$ reads $g_{1}(x)=\frac{1}{2} \sum e_{q}^{2} \Delta q(x)$, where $\Delta q(x)=$ $q^{+}(x)-q^{-}(x)$ counts the number of quarks with spin parallel to the spin of the nucleon minus the number of quarks with spin antiparallel.

In 1988 the EMC experiment measured $\Gamma_{1}=\int_{0}^{1} g_{1}(x) d x=\frac{1}{2} \sum e_{q}^{2} \Delta q$, where $\Delta q=$ $\int_{0}^{1} q(x) d x$. The sum $\Delta u+\Delta d+\Delta s$ is then the total number of quarks with spin parallel minus those with spin antiparallel, whatever their momentum and flavor, i.e. the quark spin contribution to the nucleon spin, $\Delta \Sigma$. Combining the measurement of $\Gamma_{1}$ with hyperon beta-decay data and using flavor SU(3) symmetry, they got $\Delta \Sigma=12 \pm 9 \pm 14 \%$. Due to a theoretical expectation of $\approx 60 \%$ this came as a big surprise, which was advertised as the "spin crisis", and the corresponding paper ${ }^{3}$ is still one of the 6 most cited experimental papers on the SLAC SPIRES database. The result was confirmed by SMC ${ }^{4}$, SLAC ${ }^{5}$ and Hermes ${ }^{6}$, giving $\Delta \Sigma=20$ to $30 \%$ depending on the analy- 


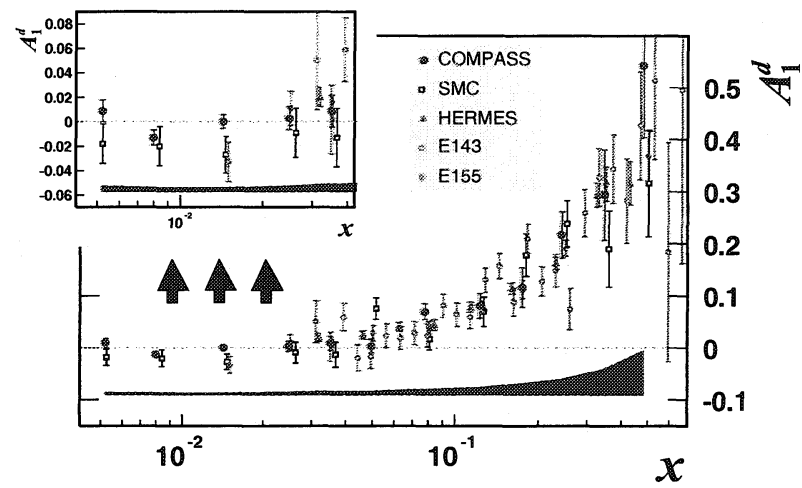

Figure 2. The virtual-photon deuteron asymmetry $A_{1}^{d} \approx g_{1} / F_{1}$ measured by COMPASS. The error bars represent the statistical error and the band the systematic error.

sis. The main uncertainty in this result arises from the extrapolation in the unmeasured region at $x<0.003$.

The new $g_{1}^{d}$ data $^{7}$ from COMPASS presented in fig 2 cover the range $0.004<x<$ 0.5. Below a few $10^{-2}$ they have much smaller errors than earlier data as can be seen in the insert. Including these data in a QCD analysis performed by COMPASS changes $\Delta \Sigma$ from $0.202_{-0.077}^{+0.042}$ to $0.237_{-0.029}^{+0.024}$, where we note a reduction of the error by about a factor 2.

The final $g_{1}$ data of Hermes ${ }^{8}$ on proton, deuteron and neutron, presented in Fig. 3, exhibit a very good statistical accuracy. The smearing between $x$ bins, due to spectrometer resolution and radiative effects, was taken into account in the analysis, resulting in a correlation between the measurements in the different bins.

Jefferson laboratory has obtained the first accurate virtual-photon neutron asymmetries $A_{1}^{n} \approx g_{1}^{n} / F_{1}^{n}$ at high $x^{9}$, showing for the first time that $A_{1}^{n}$ becomes positive for $x>0.5$. Combined with world averaged $A_{1}^{p}$ data, this provides a measurement of $\Delta u / u$ and $\Delta d / d$ as illustrated in Fig. 4. If orbital angular momentum is neglected, perturbative QCD predicts that both $\Delta u / u$ and $\Delta d / d$ should go to 1 when $x$ goes to 1 . In the figure we note that this seems indeed to

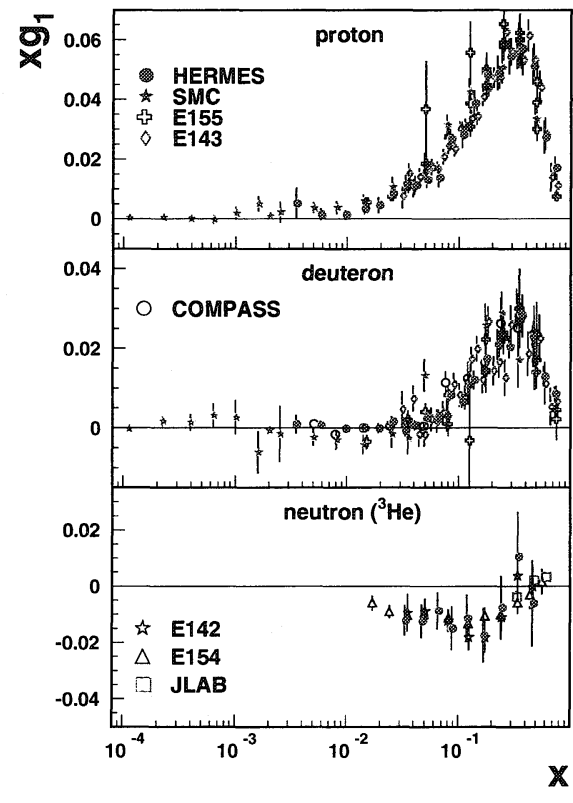

Figure 3. Caption for the figure will come here. 


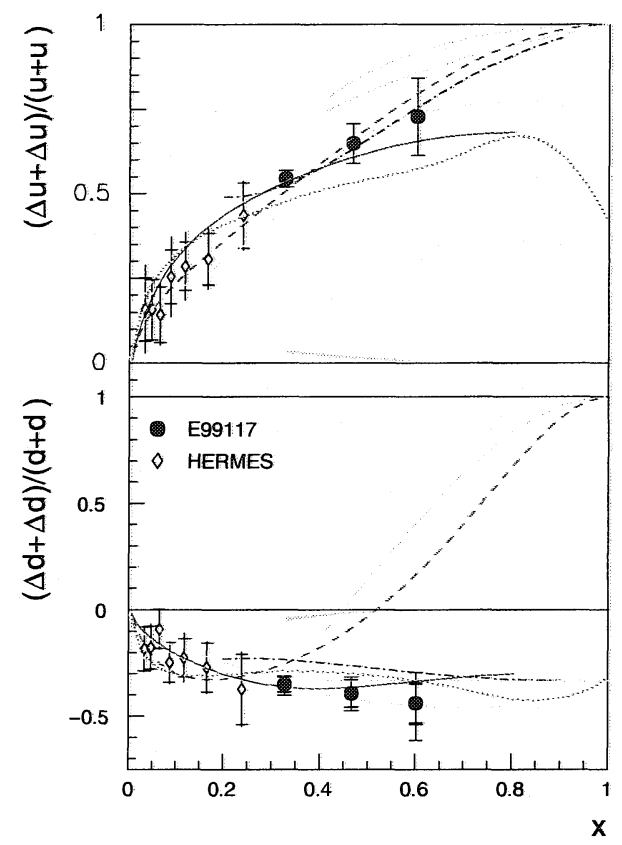

Figure 4. deltau $/ u$ and $\Delta d / d$ as a function of $x$.

be the case for $\Delta u / u$ but that $\Delta d / d$ remains negative up to the highest measured $x, 0.6$. This might indicate that orbital angular momentum cannot be neglected, at least in the particular domain of high $x$.

As a conclusion of this section devoted to quark contributions, we should say that the measurement of $\Gamma_{1}$ does not exactly provide $\Delta \Sigma$, but rather the singlet axial matrix element $a_{0}$. Naively, the two quantities are identified. However, due to the axial anomaly in QCD, they are related by

$$
a_{0}=\Delta \Sigma-\frac{3 \alpha_{s}}{2 \pi} \Delta G .
$$

If $\Delta G=0$ then $\Delta \Sigma=a_{0} \approx 0.2$, but if $\Delta G$ is large, on the order of 2.5 , we get $\Delta \Sigma$ on the order of 0.6 , which solves the spin crisis.

\section{Gluon polarized distribution}

\subsection{With a lepton beam}

Although they do not carry an electric charge the gluons can be probed with a lepton beam using the photon gluon fusion (PGF) process, $\gamma^{*} g \rightarrow q \bar{q}$, where the gluon interacts with the virtual photon through the exchange of a quark and a $q \bar{q}$ pair is produced in the final state. The cross section for this process is of course much smaller than for the leading order absorption of the virtual photon by a quark. Therefore a tagging is needed.

The cleanest tagging is provided by requiring the $q \bar{q}$ pair to be a $c \bar{c}$ pair, since the presence of $c$ quarks inside the nucleon (intrinsic charm) is negligible. The $c$ quark is identified in the form of a $D^{0}$ meson which decays to $K \pi$ with a $4 \%$ branching ratio. Due to multiple scattering inside the thick polarized target the $D^{0}$ decay vertex cannot be distinguished from the primary vertex and the $D^{0}$ is seen as a peak in the reconstructed $K \pi$ mass above a combinatorial background. This background can be reduced by selecting $D^{0}$ coming from the decay $D^{*} \rightarrow D^{0} \pi_{s}$. Because the difference of masses $M_{D^{*}}-M_{D^{0}}-M_{\pi}$ is only $6 \mathrm{MeV}$ the $\pi$ is soft and there is little phase space for the background. Due to the background and the small cross section, this channel is statistically limited: using 2002 and 2003 data COMPASS gets $\Delta G / G=-1.08 \pm 0.76$. There is twice as much data in the 2004 run and the data taking is resuming in 2006 with an improved apparatus.

In the absorption of the virtual photon by a quark, the produced hadrons go in the direction of the virtual photon with a small transverse momentum $p_{t}$ relative to it. An alternative tagging of $\mathrm{PGF}$ is then obtained by requiring a pair of hadrons with large $p_{t}$. However, there are several sources of physical background and the measured asymmetry can be written as $A_{\|}=$ $R_{P G F} a_{L L}^{P G F}(\Delta G / G)+A_{b c k g}$. Here $R_{P G F}$ is the fraction of PGF event in the sample; $a_{L L}^{P G F}$ is the analyzing power, i.e. the spin asymmetry of the PGF process which can be computed in perturbative QCD; and $A_{b c k g}$ is the asymmetry of the background. The back- 
ground includes the QCD Compton process, $\gamma^{*} q \rightarrow q g$, and at low $Q^{2}$ the resolved photon processes where the photon fluctuates to a hadronic state and one of the partons from the photon interacts with one of the partons from the nucleon.

Analyzing 2002 and 2003 data at $Q^{2}<1$ $\mathrm{GeV}^{2}$, using Pythia to estimate $R_{P G F}$ and $A_{b c k g}$, COMPASS obtains ${ }^{10}$ :

$$
\frac{\Delta G}{G}(x=0.10)=0.024 \pm 0.089 \pm 0.057 \text {. }
$$

Such a low systematic error is obtained due to the fact that a large part of the error is proportional to the asymmetry, which is small. This result is presented in Fig. 5, together with earlier measurements. The Hermes measurement ${ }^{11}$ is dominated by low $Q^{2}$ data where the resolved photon contribution is important. The background asymmetry was however neglected in this analysis, which may cast some doubt on the result. The SMC result ${ }^{12}$ and the COMPASS result at $Q^{2}>1$ come from a region where the background is easier do deal with. However, they suffer from low statistics.

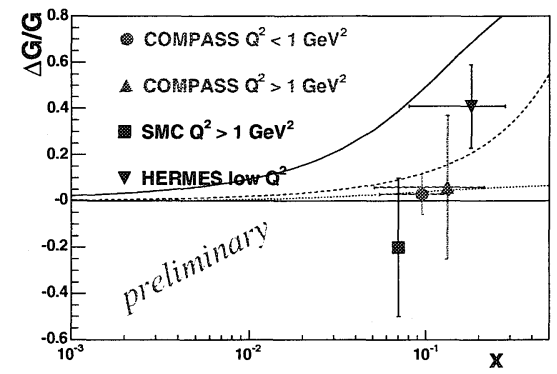

Figure 5. Various measurements of $\Delta G / G$ compared to three parametrisations from GRSV corresponding to integrals $\Delta G=\int_{0}^{1} \Delta G(x) d x=0.2,0.6$ and 2.5.

The experimental results are compared to three parametrisations from GRSV ${ }^{13}$ corresponding to integrals $\Delta G=\int_{0}^{1} \Delta G(x) d x=$ $0.2,0.6$ (standard) and $2.5(\max )$. We can see that the new COMPASS result is not compatible with the "max" parametrization with
$\Delta G=2.5$. This favors lower values of $\Delta G$, but it is still possible that $\Delta G(x)$ has a more complicated shape, e.g. crossing zero around $x=0.1$.

\subsection{With a pp collider}

The gluon polarization can also be measured in a polarized proton-proton collider, such as RHIC. The golden channel is $p p \rightarrow \gamma+$ jet $+X$. This occurs through the partonic process $q g \rightarrow q \gamma$, so it provides a convolution of $\Delta G$ in one proton with $\Delta q$ in the other proton. There is a physical background, $q \bar{q} \rightarrow$ $\gamma g$, but its contribution is small in RHIC kinematics. RHIC has not yet accumulated enough luminosity to use this channel.

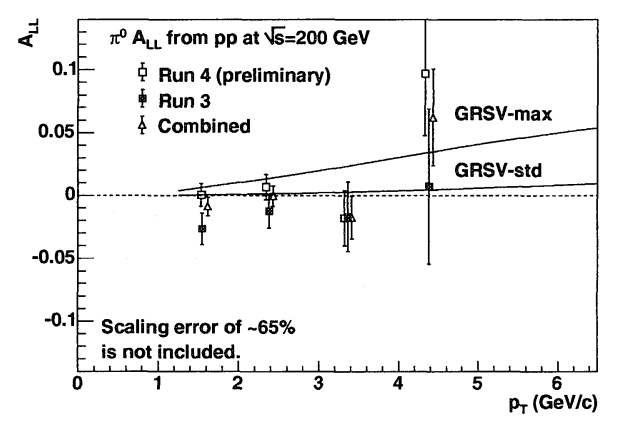

Figure 6. Longitudinal spin asymmetry $A_{L L}$ for $\vec{p} \vec{p} \rightarrow \pi^{0} X$ measured by Phenix.

Another possibility is to look at $\pi^{0}$ production. At the partonic level this involves $g g \rightarrow g g, g q \rightarrow g q$ and $q q \rightarrow q q$. So the measured asymmetry includes contributions proportional to $\Delta G \otimes \Delta G, \Delta q \otimes \Delta G$ and $\Delta q \otimes \Delta q$. Fig. 6 presents the results obtained ${ }^{14}$ by the Phenix collaboration at RHIC out of runs 3 and 4, compared to two of the previous GRSV parametrizations ${ }^{13}$. The error bars are still large but the result tends to disfavor the max parametrization. Run 5 was finished in June 2005 and it is expected to provide a gain by a factor $\approx 100$ in terms of the factor or merit, $\mathcal{L} P^{4}$. 


\section{Transversity}

At leading twist there exist three parton distribution functions for the nucleon ${ }^{15}$. The unpolarized pdf, $q(x)$, the longitudinally polarized pdf or helicity pdf, $\Delta q(x)=q^{+}(x)-$ $q^{-}(x)$, and the transversity pdf, $\Delta_{T} q(x)=$ $q^{\uparrow \uparrow}(x)-q^{\uparrow \downarrow}(x)$. Here $q^{\uparrow \uparrow}(x)$ is the probability density function for finding a quark with spin parallel to the spin of the nucleon but when the direction of the spin is observed perpendicularly to the momentum. Because rotations do not commute with Lorentz boosts transversity and helicity distributions differ. For instance, due to gluons having spin 1, there is no gluon transversity distribution, while there is a gluon helicity distribution.

Transversity is a chiral odd function. Therefore, since all hard processes conserve helicity, it decouples from DIS as illustrated in Fig. 7. In order to measure transversity, one needs to introduce a second soft object in addition to transversity, to allow for helicity flip. The simplest solution is to introduce a second proton and to measure Drell-Yan process, $p p \rightarrow l^{+} l^{-} X$. This happens through the hard process $q \bar{q} \rightarrow \gamma^{*} \rightarrow l^{+} l^{-}$and provides the convolution of $\Delta_{T} q$ in one proton by $\Delta_{T} \bar{q}$ in the other proton.

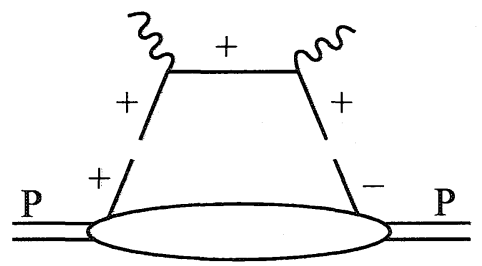

Figure 7. A pdf represents the probability to emit a parton from the nucleon. The square of the corresponding amplitude is then the lower part of the diagram. In the case of transversity the helicity of the emitted and reabsorbed partons are opposite whereas all hard processes involved e.g. in DIS conserve helicity. So the hard (upper) part of the diagram cannot be connected to the soft (lower) part, i.e. transversity cannot be measured in DIS.

It is also possible to measure transversity in semi-inclusive DIS. In this case the second soft object is a polarized fragmentation function and one measures the product of transversity times this polarized fragmentation function. Two kinds of polarized fragmentation have been used so far. The Collins fragmentation function tells how much the transverse spin of the fragmenting quark is reflected in the azimuthal distribution of the produced hadrons. It results in an azimuthal asymmetry in terms of the Collins angle, $\phi_{c o l}=\phi_{h}+\phi_{s}-\pi$, where $\phi_{h}$ is the azimuthal angle of the produced hadron and $\phi_{s}$ that of the target spin. Note that an azimuthal asymmetry in terms of the Sivers angle, $\phi_{s i v}=\phi_{h}-\phi_{s}$, is also possible. It is not related to transversity but to quark momentum distribution in the plane transverse to the nucleon momentum (intrinsic $k_{T}$ ).

The so-called interference fragmentation function is due to some interference effects in the production of two hadrons. It results in an azimuthal distribution in terms of the angle $\phi_{R S}=\phi_{R}+\phi_{S}-\pi$. In order to define $\phi_{R}$ we must introduce the momentum of the two hadrons $\vec{p}_{1}$ and $\vec{p}_{2}, \vec{p}_{h}=\vec{p}_{1}+\vec{p}_{2}, \vec{R}=\vec{p}_{1}-\vec{p}_{2}$ and $\vec{R}_{\perp}$ which is the component of $\vec{R}$ perpendicular to $\vec{p}_{h}$. Then $\phi_{R}$ is the azimuthal angle of $\vec{R}_{\perp}$.

Fig. 8 presents the Collins asymmetry measured on a proton target by the Hermes collaboration ${ }^{16}$. A clear evidence for nonzero asymmetry is seen both for the production of $\pi^{+}$and $\pi^{-}$. Fig. 9 presents the Sivers asymmetry measured also by Hermes ${ }^{16}$. The asymmetry for $\pi^{-}$is compatible with zero, but for $\pi^{+}$it is clearly positive. Such a nonzero Sivers asymmetry should in some way be related with orbital angular momentum.

Fig. 10 shows the Collins and Sivers asymmetries measured by COMPASS on a deuteron target ${ }^{17}$. The data cover a wider range of $x$ than Hermes data but, in spite of statistical errors comparable to those of Hermes, no Collins nor Siver asymmetry is observed, neither for positive nor negative hadrons. This is ascribed to a possible can- 
For Publisher's use

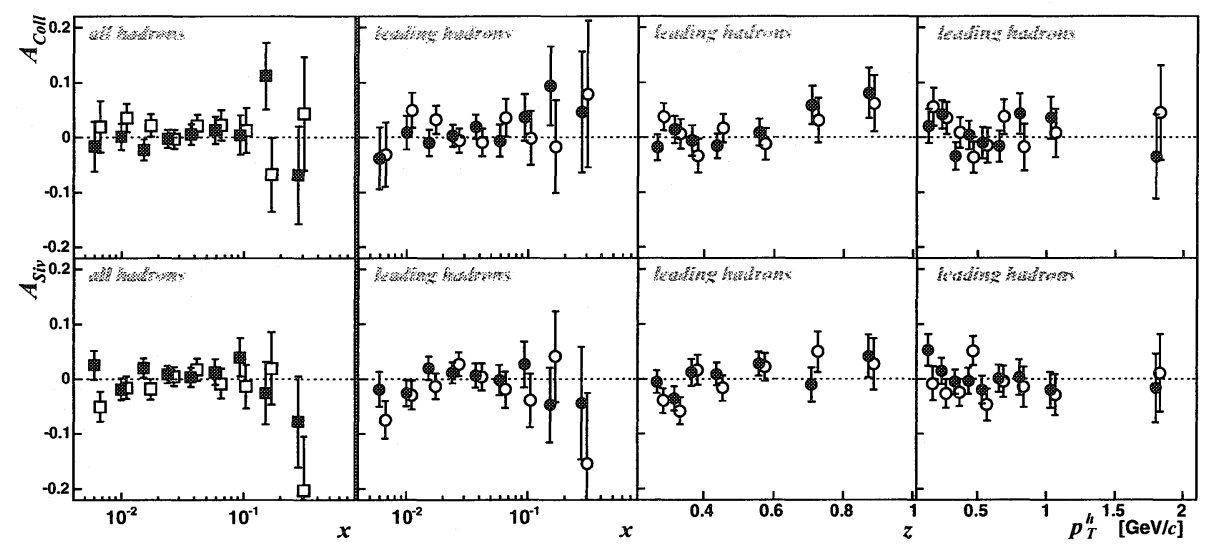

Figure 10. The Collins (upper plot) and Sivers (lower plot) asymmetries measured by COMPASS on a deuteron target for $\pi^{+}$(black squares) and $\pi^{-}$(open squares) as a function of $x, z$ and $p_{t}$.

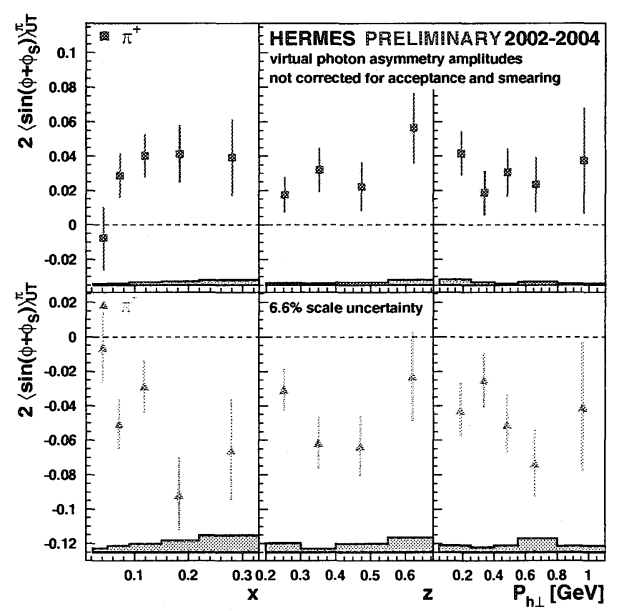

Figure 8 . The Collins asymmetry measured by Hermes on a proton target for $\pi^{+}$(upper plot) and $\pi^{-}$ (lower plot) as a function of $x, z$ and $p_{t}$.

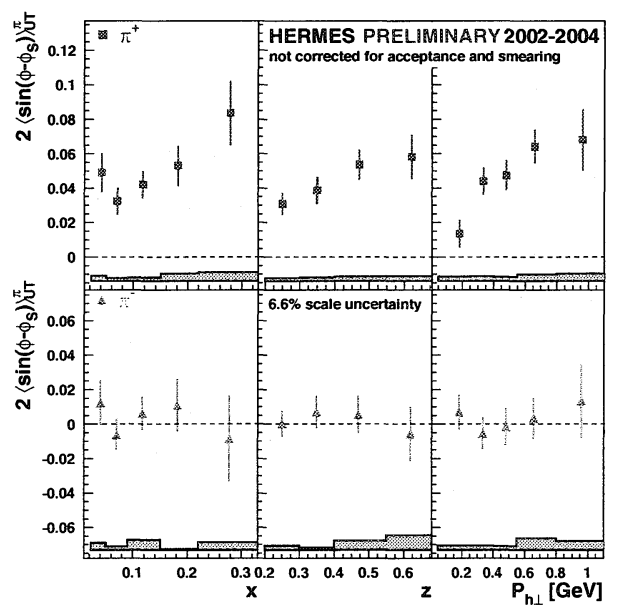

Figure 9 . The Sivers asymmetry measured by Hermes on a proton target for $\pi^{+}$(upper plot) and $\pi^{-}$ (lower plot) as a function of $x, z$ and $p_{t}$. 


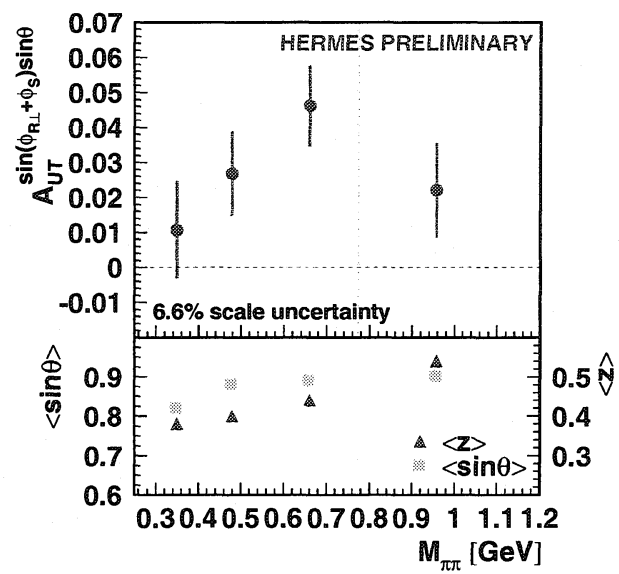

Figure 11. Interference asymmetry measured by Hermes on the proton as a function of the two pion mass. The vertical dashed line indicates the position of the $\rho$ mass.

cellation between proton and neutron asymmetries. Three times more statistics is available on tape and a run with a proton target is planned in 2006 .

The azimuthal asymmetries in terms of $\phi_{R S}$ (interference asymmetry), measured by Hermes on a proton target, is shown in Fig. 11 as a function of the two pion mass ${ }^{18}$. It clearly shows a positive asymmetry. No change of sign is observed at the $\rho$ mass in contrast with the prediction of a model by Jaffe ${ }^{19}$.

The corresponding asymmetries measured by COMPASS on a deuteron target ${ }^{20}$ are shown in Fig. 12. Once again no asymmetry is observed, probably due to a cancellation between proton and neutron asymmetries.

The Collins asymmetry, as measured By Hermes and COMPASS, is the product of transversity times a polarized fragmentation function, which is called the Collins function. This Collins function has to be measured independently. This can be done in an $e^{+} e^{-}$collider. If we consider the plane defined by the beam and jet axes, the cross section can be written as $\sigma=A+B \cos \left(\phi_{1}+\right.$

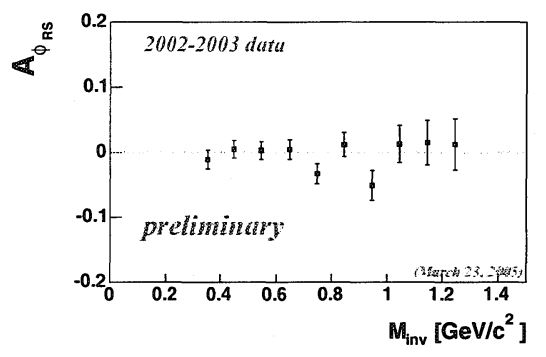

Figure 12. Interference asymmetry measured by COMPASS on the deuteron as a function of the two pion mass.
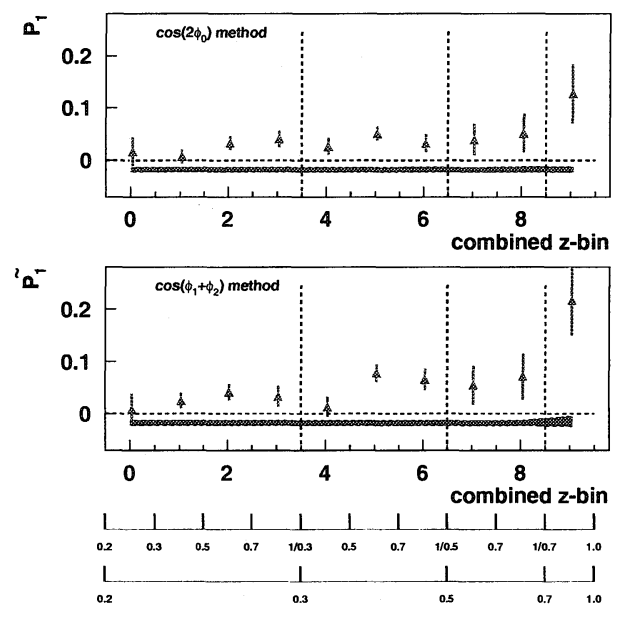

Figure 13. The azimuthal asymmetry measured by Belle as a function of $z_{1}$ and $z_{2}$.

$\left.\phi_{2}\right) \Delta_{T} D_{q}^{h}\left(z_{1}\right) \Delta_{T} D_{q}^{h}\left(z_{2}\right)$, where $\phi_{1}\left(\phi_{2}\right)$ is the azimuthal angle of the produced hadron around the axis of jet 1 (jet 2) with respect to the beam and jet plane. Hadrons $h_{1}$ and $h_{2}$ have different momentum, so $\Delta_{T} D_{q}^{h}(z)$ is probed at two different $z$.

The results obtained by Belle ${ }^{21}$ are presented in Fig. 13. The first 4 points correspond to $0.2<z_{2}<0.3$ and increasing $z_{1}$. We see a non-zero asymmetry increasing with $z_{1}$. The next 3 points correspond to $0.3<z_{2}<0.5$ and increasing $z_{1}$, we see again an asymmetry increasing with $z_{1}, \ldots$ In the last point, where both $z_{1}$ and $z_{2}$ are larger 
than 0.7 , we see a pretty large asymmetry. So we clearly see a non-zero asymmetry and it is increasing with $z_{1}$ and $z_{2}$ as expected. Ten times more statistics is available, which should allow for a deconvolution and real extraction of the Collins function as a function of $z$.

\section{Generalized parton distributions}

Generalized parton distributions ${ }^{22}$ (GPD) appear in deeply virtual Compton scattering, which is the exclusive process $\gamma^{*} p \rightarrow \gamma p$. In the deep region, i.e. large $Q^{2}$ and $-t \ll$ $Q^{2}$ where $t$ is the transfer to the nucleon, this process can be factorized at all orders in QCD. At Leading order, as illustrated in Fig. 14, a quark of longitudinal momentum fraction $x-\xi$ is emitted by the nucleon, the quark absorbs the virtual photon, emits a real photon and is reabsorbed with a momentum fraction $x+\xi$. The factorization involves new soft objects, the generalized parton distributions which are real functions of $x, \xi$ and $t$. There exist four of them, labelled $H, \tilde{H}, E$ and $\tilde{E}$.

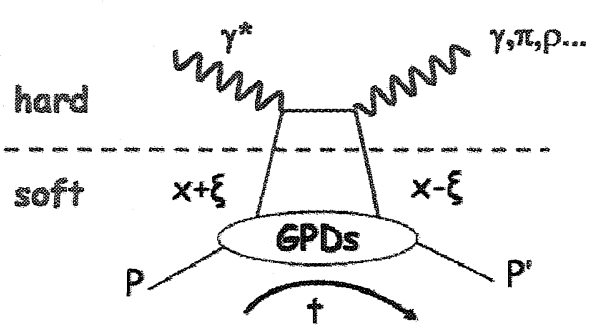

Figure 14. The DVCS diagram at leading order.

Due to the optical theorem the DIS crosssection is proportional to the imaginary part of forward Compton scattering, $\gamma^{*} p \rightarrow \gamma^{*} p$. The diagram for the DVCS amplitude is then very similar to the diagram for the DIS crosssection. The difference is that DVCS probes correlations $\Psi(x-\xi) \Psi^{*}(x+\xi)$ while DIS probes $|\Psi(x)|^{2}$ and that DVCS implies a nonzero momentum transfer to the nucleon, $t$.
Two of the GPDs correspond to the same nucleon spin in the initial and final states, in the limit $t=0$ and $\xi=0$ they give back pdf, namely $H(x, 0,0)=q(x)$ and $\tilde{H}(x, 0,0)=$ $\Delta q(x)$. The two other GPDs have $s \neq s^{\prime}$ and disconnect from the cross section in the limit $t=0$.

The GPDs are also related to the form factors $F(t)$, which are the Fourrier transforms of the spatial distributions of quarks inside the nucleon. We have the sum rules $\int H(x, \xi, t) d x=F_{1}(t)$ and $\int E(x, \xi, t) d x=$ $F_{2}(t)$, where $F_{1}$ and $F_{2}$ are the Dirac and Pauli form factors. In the limit $\xi=0$ the GPDs can be shown to provide a $3 \mathrm{D}$ view of the nucleon in terms of the momentum fraction and the impact parameter, $f\left(x, d_{\perp}\right)$. It is therefore intuitive that GPDs have something to do with the orbital angular momentum. This relation is formalized in the socalled Ji sum rule.

We can see that the DVCS diagram involves a loop over $x$. Therefore the amplitude is an integral of GPD over $x$ :

$$
\begin{gathered}
T_{D V C S} \propto \int \frac{d x}{x-\xi+i \epsilon} H(x, \xi, t)= \\
\mathcal{P} \int \frac{d x}{x-\xi} H(x, \xi, t)-i \pi H(x=\xi, \xi, t) .
\end{gathered}
$$

In addition DVCS interferes with the BetheHeitler process which is just an elastic scattering on the nucleon accompanied by the radiation of a real photon, either by the initial or the final lepton.

This interference can actually be useful. Since the Bethe-Heitler amplitude is known, the measurement of the interference provides a measurement of the DVCS amplitude. The interference can be measured in single spin asymmetry (polarized beam) which selects the imaginary part of the DVCS amplitude, i.e. $H(x=\xi, \xi, t)$; while the beam charge asymmetry $\left(e^{+}\right.$versus $\left.e^{-}\right)$selects the real part of the DVCS amplitude, i.e. $\int \frac{d x}{x-\xi} H(x, \xi, t)$.

Measurements of single spin asymmetries and beam charge asymmetries have been per- 
For Publisher's use

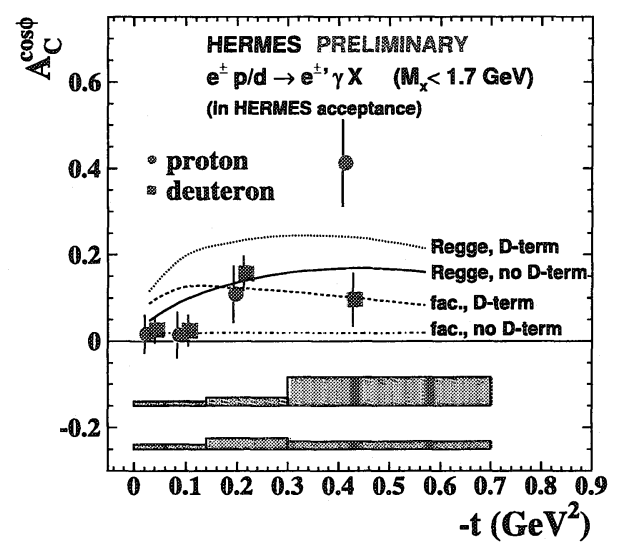

Figure 15. Beam charge asymmetry for the DVCS process as a function of $-t$ for the proton and the deuteron. Hermes data are compared to the predictions of different models of proton GPDs

formed by Jefferson Lab ${ }^{23}$ and Hermes ${ }^{24}$. Fig. 15 presents the beam charge asymmetries obtained recently by Hermes ${ }^{25}$. We see that the error bars are still too large in these exploratory data to discriminate the models. However, much more precise data are currently being analyzed at Jefferson Lab and new precise data should be taken by Hermes in the coming years. After 2010 DVCS experiments at COMPASS and at a $12 \mathrm{GeV}$ upgraded Jefferson lab should cover a wider kinematic range.

\section{Conclusions}

The E821 experiment at Brookhaven gives a muon anomalous momentum which is 2.7 $\sigma$ from the standard model prediction. A new experiment and progress in theory are expected to reduce the error by a factor 2 .

The spin structure of the nucleon is a very active field. More topics than could be discussed here are being studied, like the tensor structure function of the deuteron at Hermes.

There are some first indications that the gluon spin contribution to the nucleon, $\Delta G$, could be smaller than the value of about
2.5 which was somewhat expected because it would solve the "spin crisis". If this is indeed the case, we are left with the crisis, i.e. with a small contribution of the quark spin, $\Delta \Sigma=0.2-0.3$.

Non-zero asymmetries related to transversity have been measured by Hermes. The extraction of transversity then requires to know a polarized fragmentation function, the Collins function. Asymmetries related to the Collins function were measured in $e^{+} e^{-}$ collisions by Belle and the Collins function itself should be extracted soon.

Generalized parton distributions (GPD) represent an opening field. Exploratory measurements have been performed and new much more precise data are expected soon.

Several new projects are developing over the world. An upgraded version of COMPASS should allow for the measurements of GPD in a wide kinematical range. An upgraded Jefferson Lab at $12 \mathrm{GeV}$ will also probe GPDs. The PAX project at the $p \bar{p}$ collider at GSI will be the ideal tool to study transversity in the cleanest channel, i.e. in Drell-Yan processes. The most complete program is certainly that of the eRHIC project of a polarized electron-proton collider. This will give the possibility to reach low $x$, to have enough level arm in $Q^{2}$ for an accurate NLO analysis providing $\Delta q$ and $\Delta G$, to measure directly $\Delta G(x)$ and to further study GPDs.

\section{References}

1. Muon (g-2) Collaboration, G. W. Bennett et al, Phys. Rev. Lett. 92 (2004) 161802; David W. Hertzog and William M. Morse Annu. Rev. Nucl. Part. Sci. 54, 141-174 (2004).

2. Reviews: M. Anselmino, A. Efremov and E. Leader, Phys. Rep. 261 (1995) 1, hep-ph/9501369; B. Lampe and E. Reya, Phys. Rep. 332 (2000) 1, hep-ph/9810270

3. J. Ashman et al., EMC coll., Phys. 
Lett. B206 (1988) 364.

4. SMC, D. Adams et al., Phys. Rev. D58 (1998) 112001.

5. E142, Anthony et al. Phys. Rev. D54 (96) 6620; E143, Abe et al., Phys. Rev. D58 (98) 112003; E154, Abe et al., Phys. Lett. B405 (97) 180; E155, Anthony et al., Phys. Lett. B493 (00) 19.

6. Hermes, A. Airapetian et al., Phys. Lett. B442 (1998) 484.

7. COMPASS, E.S. Ageev et al., Phys. Lett. B612, 154 (2005).

8. Hermes: D. Reggiani, AIP Conference Proceedings 792, DIS 2005, p 913 Springer Verlag (2005).

9. X. Zheng et al., Phys. Rev. Lett. 92, 012004 (2004)

10. COMPASS: C. Bernet, AIP Conference Proceedings 792, DIS 2005, p 949 Springer Verlag (2005); hep-ex/0507049.

11. Hermes, A. Airapetian et al., Phys. Rev. Lett. 84 (2000) 2584.

12. SMC, B. Adeva et al., Phys. Rev. D70 (2004) 012002.

13. M. GLuck, E. Reya, M. Stratmann, W. Vogelsang, Phys. Rev. D63 (2001) 094005.

14. A. Deshpande, AIP Conference Proceedings 792, DIS 2005, p 1001 Springer Ver$\operatorname{lag}(2005)$.

15. Review: V. Barone, A. Drago and P.G. Ratcliffe, Phys. Rep. 359 (2002) 1; hep$\mathrm{ph} / 0104283$.

16. Hermes: A. Airapetian et al., Phys. Rev. Lett. 94, 012002 (2005); hepex/0408013.

17. COMPASS: V. Y. Alexakhin et al., Phys. Rev. Lett. 94 (2005) 202002; hepex/0503002.

18. Hermes: P.B. Van der Nat, AIP Conference Proceedings 792, DIS 2005, p 953 Springer Verlag (2005).

19. R. L. Jaffe, X. m. Jin and J. Tang, Phys. Rev. Lett. 80 (1998) 1166; hep$\mathrm{ph} / 9709322$.

20. COMPASS: R. Joosten, AIP Confer- ence Proceedings 792, DIS 2005, p 957 Springer Verlag (2005).

21. A. Ogawa et al., AIP Conference Proceedings 792, DIS 2005, p 949 Springer Verlag (2005).

22. Reviews: M. Diehl, Phys. Rep. 388 (2003) 41; A.V. Belitsky, D. Müller and A. Kirchner, Nucl. Phys. B629 (2002) 323.

23. CLAS, S. Stepanyan et al., Phys. Rev. Lett. 87 (2001) 182002.

24. A. Airapetian et al., Phys. Rev. Lett. 87 (2001) 182001, hep-ex/0106068

25. M. Kopytin, AIP Conference Proceedings 792, DIS 2005, p 949 Springer Ver$\operatorname{lag}(2005)$. 


\section{DISCUSSION}

Volker Burkert (Jefferson Lab):

If $\Delta G$ remains small does that mean that the orbital angular momentum contributions to the nucleon spin must be large? If so, does this put more emphasis on programs to measure the generalized parton distributions (GPD) to allow access to $L_{z}$ ?

Jean-Marc Le Goff: Actually I can see two levels of $\Delta G$ being small. The axial anomaly gives

$$
\Delta \Sigma=a_{0}+\frac{3 \alpha_{s}}{2 \pi} \Delta G,
$$

with $a_{0} 0.2$ to 0.3 . If $\Delta G$ is on the order of 2 or 3 then we get $\Delta \Sigma$ on the order of 0.6 as expected, which "solves the spin crisis". So the first level of being small is relative to the large values of 2 or 3. And the data tend to disfavor such a scenario, even if they cannot exclude it completely.

On the other hand we have the momentum sum rule

$$
\frac{1}{2}=\frac{1}{2} \Delta \Sigma+\Delta G+L_{q}+L_{g} .
$$

Here, with $a_{0} 0.2$ to 0.3 , we need $\Delta G \approx$ 0.4 to fill the sum rule. So the other level of being small is relative to this value of 0.4 . And according to the current data a value of 0.4 is as likely as a null value. So I do not think that the current $\Delta G$ data can be used to claim that $L_{z}$ should be important.

However, there are several indications that $L_{z}$ should not be neglected. I mentioned in the talk the observation of a non-zero Sivers asymmetry and also the finding that $\Delta d / d$ is negative at high $x$, which both point to orbital angular momentum. In addition GPDs are a very interesting topic besides the issue of $L_{z}$. They generalized the notion of pdf and form factor, providing a $3 \mathrm{~d}$ view of the nucleon. So I think we have very strong motivations to study GPD and I am looking forwards seeing the new accurate Jefferson lab DVCS data. 